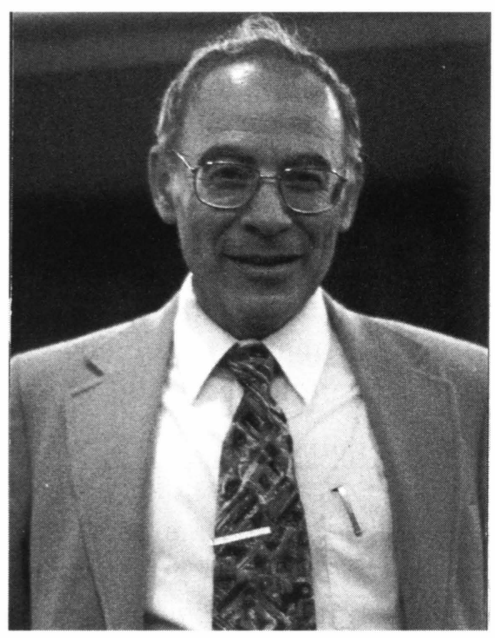

\title{
Reflections on the Future of Mathematics
}

\section{by Avner Friedman}

Imagine Archimedes (287-212 BC), one of the most distinguished mathematicians of all times, being asked: What do you see in the future for mathematics? Perched on the beach of his hometown, Syracuse, on the eastern shore of Sicily, the ancient mathematician, having just computed the surface and volume of spheres, or the area of a parabolic section, straightens up his back and looks intently into the horizon. He wonders: What else could he or anyone else do with mathematics? One of his great ambitions was to be able to compute volumes and surfaces of any geometric object; yet he had no idea how to go about it. His tools were purely geometrical, based on several hundred years of research by Greek mathematicians, edited several decades before his birth by Euclid in his famous book, Elements.

Being quite limited in his mathematical tools, Archimedes was undoubtedly also limited in his vision. He did not have a quick way of adding or multiplying fractions. For it would take more than a thousand years before the decimal system would be developed and transported to Europe by the Hindus and Arabs. The simplification of notation introduced by the decimal system was revolutionary in its enabling power.

Leaving Archimedes on the sands of Syracuse to reflect on what else is there in the future for mathematics, we now visit with Sir Isaac Newton (1642-1727). At 23, having just received his B.A. from Cambridge University, Newton was forced to return home for 18 months when the Great Plague caused the closure of the University. During this short period, Newton made several fundamental discoveries: In mathematics he discovered the binomial theorem and the early form of differential and integral calculus, and in physics he discovered the composition of white light and the universal law of gravity. We are now meeting Newton at an advanced age and asking him the same question we posed to Archimedes: What is the future for mathematics?

The easy answer, he could quickly respond, is to continue to build on differential and integral calculus. By means of calculus, Newton could express the surface or volume of any geometric shape by integrals and could then compute them to any degree of accuracy, something Archimedes could not possibly have imagined. Newton reflects on the fact that with the law of gravity and his own three fundamental laws of mechanics ("my three laws", he might say), he can compute trajectories of moving objects by solving a differential equation which expresses a balance of forces. "Can we describe other laws of nature by differential equations, and can we then predict nature's course by developing tools to solve those equations?" he wonders.
But even Newton's vision is inevitably limited. It will take a hundred years before Gauss (1777-1855) will make his fundamental discoveries in number theory, and fifty more years before Riemann (1826-1866) will develop the intricate nature of differential geometry and Riemannian manifolds.

As we move closer to the present, the future becomes easier to predict. David Hilbert (1862-1943), a substantial contributor in almost every field of mathematics, formulated his famous set of "mathematical problems" in the International Mathematical Congress in Paris (1900). This has had a great impact throughout the 20th century on diverse mathematical fields such as number theory, set theory, geometry, topology, and partial differential equations.

In the last fifty years, we have witnessed great strides in many fields of mathematics. In Partial Differential Equations (PDE) in which I work, we now have a vast body of knowledge which enables us to understand, predict, and compute many important physical and technological processes. If we measure, for example, the temperature on the surface of a solid body, then we can infer the temperature inside the body by solving a partial differential equation, called the "heat equation." If a body of ice, heated from the outside, begins to melt, our knowledge in differential equations enables us to determine how the melted volume will grow and what the water temperature will be inside the melted volume. The "beam equation" similarly predicts how elastic beam will change shape when subjected to compressive stress. When the pressure on the beam exceeds a critical value the beam will suddenly buckle and deform into one of several states. This situation illustrates multiplicity of solutions provided by differential equations.

Yet in spite of our extensive body of knowledge in differential equations, there are many things we do not know. We do not know, for example, whether 
the equations of gas dynamics, which determine the air flow around airplanes or in their engines, have a mathematical solution; we do not have adequate knowledge regarding the behavior of solutions to the equations that predict the motion of water and, consequently, we lack understanding of turbulent flow in the ocean. These fundamental problems and many others still await their mathematical solutions; they will continue to be the subject of intensive research in the coming decades.

Other fields of mathematics are undoubtedly in the same tentative state: Although vast progress has been made, many fundamental problems remain unanswered. Compared to earlier centuries, we are in an adventurous position: We have developed many important research areas and have many powerful computational and theoretical tools. Mathematicians can be kept busy for many years to come by developing new ways of applying the present tools to solve problems that arise both within mathematics and without (i.e., in the sciences and engineering).

However, the history of mathematics shows how futile it is to predict long term future discoveries from the present. Indeed, new fields of mathematics, unimaginable today, may arise quite unexpectedly.

Thus instead of predicting the future of mathematics for the next century, I shall offer here three examples of key fields in science and technology where mathematics is emerging as a vital component: Materials sciences, the life sciences, and digital technology.

(i) Mathematics in Materials Sciences. Materials sciences is concerned with the properties and the use of materials. The objectives are the synthesis and manufacture of new materials, the modification of materials, the understanding and prediction of material properties, and the evolution and control of these properties over a time period. Until recently, material sciences was primarily an empirical study in metallurgy, ceramics, and plastics. Today it is a vast growing body of knowledge based on physical sciences, engineering, and mathematics. Properties of all materials ultimately depend on their atoms and on their grouping into molecular structure. Polymers, for example, are substances composed of simple molecules that are repeated structural units, called monomers. A single polymer molecule may consist of hundreds to a million monomers and have a linear, branched or network structure.

Polymeric material may appear in either fluid or solid form, and its properties depend on the manner in which it was processed (e.g., fast heating, slow cooling, high pressure). The entanglement of polymers poses difficult modeling problems. However, there are already some areas where mathematical models are emerging as quite reliable. Some of these models are based on statistics or statistical mechanics; others are based on a diffusion equation in finite or infinite dimensional spaces. These models are very complicated, so only very few results have been obtained so far that may be useful to the processing of polymers. Simpler but more phenomenological models of polymers are based on Continuum Mechanics with added terms to account for "memory." Stability and singularity of solutions are important issues for materials scientists, yet the mathematics is still lacking even for these simpler models.

The study of composites is another emerging area using mathematical research. If we insert into one material grains of another material, we get a composite material which may exhibit properties that are radically different from those of its constituents. Automobile companies, for example, are working with composites of aluminum and silicon-carbon grains which provide light-weight alternative to steel. Fluid with magnetic particles or electrically charged particles will enhance the effects of brake fluid and shock absorbers in the car.

Over the last decade, mathematicians have made important discoveries in the study of composites. They have developed new tools in functional analysis, PDE, and numerical analysis, by which they have been able to estimate or compute the effective properties of composites. But the list of new composites is ever increasing and new materials are constantly being developed (e.g., "shape memory", a substance, if folded or distorted in any other way, will always return to its orginal shape when heated). Considering the vast demand for understanding and analysis of these new materials, the mathematical achievements up to now may be viewed only as a very modest beginning.

Mathematical challenges abound even for standard materials which have been studied for some years. For example, when a uniform elastic body is subjected to high pressure, cracks will form. Where and how the cracks initiate, how they evolve, and when they branch out into several cracks are questions that are still being researched.

(ii) Mathematics in Biology. Mathematical models are also emerging in the biological and medical sciences. The highly publicized genome project has important aspects which require statistics, pattern recognition, and large-scale optimization methods. Less publicized but longer-term challenges are developing in other areas of biology such as physiology. Take, for instance, the kidney, whose function is to 
regulate the composition of the blood by maintaining a desired level of concentration of critical substances like salt. If a person consumes too much salt, the kidney will have to excrete urine in which the salt concentration is greater than in the blood. One million tiny tubes around the kidney, called nephrons, have the task of absorbing salt from the blood into the kidney. They do it through contact with blood vessels by a transport process in which osmotic pressure and filtration play a role. Biologists have identified the body tissues and substances which are involved in this process, but the precise rules of the process are only barely understood. An initial mathematical model of the renal process, simple as it is, is already shedding some light on the formation of urine and on decisions made by the kidney on whether, for example, to excrete a large volume of diluted urine or a small volume of concentrated urine. However, we are only at the very early stage of understanding of this mechanism. A more complete model may include PDE, stochastic equations, fluid dynamics, elasticity theory, filtering theory, and control theory, and perhaps other tools that we do not yet have.

Heart dynamics, calcium dynamics, the auditory process, cell adhesion and motility (vital for physiological processes such as inflammation and wound healing) and biofluids are other topics in physiology where recent mathematical studies have already made some progress; much more is still to come.

Other areas where mathematics is poised to make important progress include the growth process in general and embryology in particular, cell signaling, immunology, emerging and re-emerging infectious diseases, and ecological issues such as global phenomena in vegetation and modeling animal grouping. And, of course, we must not forget the human brain, nature's best computer, with all its complex sensors and actuators and emotions and dreams!

Biological processes are inherently very complex, and the mathematical challenges will undoubtedly occupy researchers for many decades, perhaps centuries.

(iii) Mathematics in Multimedia. About fifty years ago the first computer was built. Thus began a quiet revolution, similar in scope to the industrial revolution that took place in England from 1760 to 1840 . We are now witnessing the full impact of the computer revolution in business, manufacturing, health services, and engineering. Industry is making a substantial investment to deliver digital audio, image, and video information to consumers. The burgeoning of digital information, coupled with advances in computing and communication technologies, has paved the way for multimedia, whose products may contain graphics, film, video, music, photographs, painting, animation, data, games and multimedia software, all delivered on a single platform.
The mathematics of multimedia encompasses a wide range of research areas which include computer vision, image processing, speech recognition and language understanding, computer aided design, and new modes of networking. These will have a wide spectrum of applications in manufacturing, business, and banking industries, medical diagnostics, information and visualization, and in the entertainment industry, to name a few. The mathematical tools in multimedia may include stochastic processes, Markov fields, statistical patterns, decision theory, PDE, numerical analysis, graph theory, graphic algorithms, image analysis and wavelets, and many others, some of which would seem presently somewhat escoteric, such as artificial life and virtual reality.

Many challenges face us in the ever increasing demand to compress, store, and then retrieve information, especially because visual information requires a huge amount of data.

Computer aided design is becoming a powerful tool in many industries: Products, designed entirely on the computer, materialize with a tap on the keyboard, on factory floors some distance away. Could this technology become a tool in the hands of research mathematicians? The world wide web (WWW) has become the most powerful engine for multimedia technology. Its future exploits will depend on the development of many new mathematical ideas and algorithms, presently in their infancy. As multimedia technologies expand, the need to develop communication protocols that protect data privacy will grow. It will be the task of mathematicians to develop ever more secure cryptographic schemes. In doing so, they will undoubtedly draw upon new developments from number theory, discrete mathematics, algebraic geometry, and dynamical systems, as well as other fields.

Mathematics will continue to play an ever-increasing role in the development of the physical and life sciences and in technology; because of this, its core will also be enriched and new fields will emerge. Like Archimedes on the beach of Syracuse, here we stand on the threshold of a new century and a new millennium. We can only speculate about the new theories that will eventually solve the challenging mathematical problems that arise from the world in which we live, as well as within mathematics itself. In the last few centuries we have gained a tremendous amount of knowledge, but like Archimedes and Newton we are still on the threshold of ever expanding mathematical horizons.

\section{The author's address:}

Prof. Avner Friedman

Institute for Mathematics and its Applications University of Minnesota 